OPEN ACCESS

Edited by:

Alla B. Salmina,

Krasnoyarsk State Medical University

named after Prof.

V.F.Voino-Yasenetski, Russia

Reviewed by:

Henning U. Voss,

Cornell University, United States

Hirohisa Watanabe,

Fujita Health University, Japan

Martin J. McKeown,

University of British Columbia,

Canada

Alle Meije Wink,

VU University Medical Center,

Netherlands

*Correspondence:

Jianguo Zhong

zjg_zjsrmyy@126.com

Received: 22 April 2020 Accepted: 11 September 2020

Published: 16 October 2020

Citation:

Cao F, Guan X, Ma Y, Shao Y and Zhong J (2020) Altered Functional Network Associated With Cognitive

Performance in Early Parkinson

Disease Measured by Eigenvector

Centrality Mapping.

Front. Aging Neurosci. 12:554660.

doi: 10.3389/fnagi.2020.554660

\section{Altered Functional Network Associated With Cognitive Performance in Early Parkinson Disease Measured by Eigenvector Centrality Mapping}

\author{
Fang $\mathrm{Cao}^{1}$, Xiaojun Guan ${ }^{2}$, Yanqing $\mathrm{Ma}^{1}$, Yuan Shao ${ }^{1}$ and Jianguo Zhong ${ }^{1 *}$ \\ ${ }^{1}$ Department of Radiology, Zhejiang Provincial People's Hospital, People's Hospital of Hangzhou Medical College, Hangzhou, \\ China, ${ }^{2}$ Department of Radiology, Second Affiliated Hospital, School of Medicine, Zhejiang University, Hangzhou, China
}

Objective: To investigate relationships between whole-brain functional changes and the performance of multiple cognitive functions in early Parkinson's disease (PD).

Methods: In the current study, we evaluated resting-state functional MRI (rsfMRI) data and neuropsychological assessments for various cognitive functions in a cohort with 84 early PD patients from the Parkinson's Progression Markers Initiative (PPMI). Eigenvector centrality $(E C)$ mapping based on rsfMRI was used to identify the functional connectivity of brain areas correlated with different neuropsychological scores at a whole-brain level.

Results: Our study demonstrated that in the early PD patients, scores of Letter Number Sequencing (LNS) were positively correlated with EC in the left inferior occipital gyrus (IOG) and lingual gyrus. The immediate recall scores of Hopkins Verbal Learning Test-Revised (HVLT-R) were positively correlated with EC in the left superior frontal gyrus. No correlation was found between the EC and other cognitive performance scores.

Conclusions: Functional alternations in the left occipital lobe (inferior occipital and lingual gyrus) and left superior frontal gyrus may account for the performance of working memory and immediate recall memory, respectively in early PD. These results may broaden the understanding of the potential mechanism of cognitive impairments in early PD.

Keywords: Parkinson disease, fMRI, eigenvector centrality, cognition, memory, executive function

\section{INTRODUCTION}

Cognitive impairment is a serious and common nonmotor symptom of Parkinson's disease (PD), which has received increasing attention in recent years. It can occur at different stages of PD, even at the time of PD diagnosis (Williams-Gray et al., 2007). In patients newly diagnosed with PD, the frequency of mild cognitive impairment (MCI) was approximately $20-40 \%$ (Yarnall et al., 2014; Aarsland et al., 2017). Early MCI is an important risk factor for dementia. The manifestations of cognitive decline in PD can be highly heterogeneous. Dysfunction in various cognitive domains has 
been reported in the early stages of PD before overt dementia, such as executive functions, memory, attention, visuospatial performance, and language (Yarnall et al., 2014; Aarsland et al., 2017). A previous study suggested that deficits in specific cognitive functions imposed different degrees of risk for developing dementia (Pagonabarraga and Kulisevsky, 2012). However, the potential brain mechanisms of dysfunction in the processes of diverse cognitive functions remain to be fully studied.

Several MRI studies have explored brain structural and functional network changes related to cognitive performance in PD patients. Widespread gray matter atrophy or white matter damage was observed in PD patients with relatively long disease duration (Melzer et al., 2012; Agosta et al., 2014). A few studies based on structural MRI further suggested that differences in regional brain volume may contribute to between-patient cognitive heterogeneity in PD (Gerrits et al., 2016; Gao et al., 2017). However, in the early stages of PD, structural changes were always limited and subtle. Functional MRI (fMRI) studies indicated that functional alternations in widely distributed brain networks, such as the frontoparietal network, cortico-striatal circuits, default mode network (DMN), were associated with overall cognitive decline in patients with PD (Monchi et al., 2007; Tessitore et al., 2012; Díez-Cirarda et al., 2018; Hou et al., 2018). Most of the previous fMRI studies investigated functional changes in a priori seed regions or subnetworks. Moreover, to our knowledge, the neuroanatomic basis of dysfunction in some specific cognitive processes was shown to be different between PD and other neurodegenerative diseases (Lee et al., 2010). The key brain nodes or networks related to deficits in various cognitive functions in early $\mathrm{PD}$ remained to be illuminated. Therefore, a broader and more integrated brain network beyond the known focal regions or subnetworks needs to be investigated.

As a data-driven approach, graph theory-based methods have been increasingly proposed for the analysis of brain MRI data and became a powerful tool for exploring alterations in brain functional and structural networks. Brain networks are described as graphs composed of nodes (anatomic brain regions) linked by edges (i.e., functional connections). To our knowledge, only a few studies used graph theory-based methods to evaluate cognitive impairment in PD patients (Baggio et al., 2014). In the present study, we focus on a special type of graph theory-based method that identifies nodes playing central roles within the brain network. Such nodes are characterized by a measure called "centrality". Eigenvector centrality (EC) measures the sum of centralities of a node's direct neighbors by assessing both the number and the quality of connections, making it sensitive to different layers in the network hierarchy (Lohmann et al., 2010; Wink et al., 2012). EC mapping (ECM) attributes a value of each voxel in the whole brain, therefore, it can objectively detect all the important brain regions that have greater connections with other parts of the brain. Unlike seed-based analysis or Independent Component Analysis (ICA), EC considers the brain as one large network rather than several subnetworks, so it is independent of a priori knowledge and free of researcher selection bias. ECM has proven valuable in investigating various nervous system diseases, such as Alzheimer's disease (AD), major depressive disorder, and nicotine dependence (Binnewijzend et al., 2014; Song et al., 2016; Shen et al., 2017).

In the present study, we aimed to investigate the alterations of functional connections that were associated with the performance of multiple cognitive functions over the entire brain in early $\mathrm{PD}$ patients by using EC as a mapping tool.

\section{MATERIALS AND METHODS}

\section{Subjects}

Data used in this article were obtained from the Parkinson's Progression Markers Initiative (PPMI) database ${ }^{1}$. PPMI is an observational, multi-center study that has been collecting a large variety of clinical, imaging data and biologic samples to identify more biomarkers of PD progression. This research protocol was approved by local Institutional Review Boards of all the participating institutions, and written informed consent was obtained from all participants. Major inclusion criteria of early PD subjects were as follows: (1) at least two of the following symptoms: resting tremor, bradykinesia, rigidity or either asymmetric resting tremor or asymmetric bradykinesia; (2) Hoehn and Yahr stage I or II; (3) age 30 years or older; and (4) dopamine transporter deficit suggested by dopamine transporter SPECT scan. Exclusion criteria mainly included atypical PD syndromes and any medical or psychiatric condition or lab abnormality, which might preclude participation.

\section{Neuropsychological Assessments}

Participants underwent a series of cognitive tests evaluating various cognitive functions, including: (1) Hopkins Verbal Learning Test-Revised (HVLT-R) as a measure of verbal memory; (2) Benton Judgment of Line Orientation (BJLO) test for assessing visuospatial function; (3) Semantic Fluency test mainly for reflecting executive function; (4) WMS-III Letter Number Sequencing (LNS) test as a measure of working memory; and (5) Symbol Digit Modalities Test (SDMT) for assessing attention function.

\section{Imaging Data Acquisition}

Both structural and rest-state fMRI (rsfMRI) data were downloaded from the PPMI database. A standard MR scan was performed using a Siemens 3T TIM Trio scanner. The structural images were obtained using a 3D MPRAGE with GRAPPA T1-weighted sequence: sagittal orientation; repetition time $(\mathrm{TR})=2,300 \mathrm{~ms}$; echo time $(\mathrm{TE})=2.98 \mathrm{~ms}$; inversion time $(\mathrm{TI})=900 \mathrm{~ms}$; flip angle $(\mathrm{FA})=9^{\circ}$; Field of View $_{(\mathrm{FOV})}=240 \times 256 \mathrm{~mm}^{2}$; voxel size $=1 \times 1 \times 1 \mathrm{~mm}^{3}$. The rsfMRI images were acquired using an echo-planar imaging sequence with following parameters: 185 EPI volumes, with 40 ascending slices each; $\mathrm{TR}=2,400 \mathrm{~ms}$; $\mathrm{TE}=25 \mathrm{~ms}$; flip angle $=80^{\circ}$; slice thickness $=3.3 \mathrm{~mm}$ FOV $=222 \times 222 \mathrm{~mm}^{2}$; voxel size $=3.3 \times 3.3 \times 3.3 \mathrm{~mm}^{3}$.

\footnotetext{
${ }^{1}$ www.ppmi-info.org/data
} 


\section{Imaging Preprocessing}

Image data were preprocessed with the Data Processing and Analysis for Brain Imaging ${ }^{2}$, based on the Statistical Parameter Mapping software (SPM $8^{3}$ ). The first 10 volumes were discarded from the analysis. The remaining rsfMRI images were used for the following procedures: slice timing correction (using middle slice as a reference) and realignment to correct head motion, nuisance covariates regression (Friston 24 parameters, white matter, and cerebrospinal fluid signal), normalization to the standard space and resampling into a $3 \times 3 \times 3 \mathrm{~mm}^{3}$ voxel size, spatially smoothing with a Gaussian kernel of $6 \mathrm{~mm}$ full width at half maximum (FWHM), temporal band-pass filtering $(0.01-0.1 \mathrm{~Hz})$, detrending and scrubbing. Subjects were excluded if the head motion was exceeding $2 \mathrm{~mm}$ (displacement in the $\mathrm{x}$, $\mathrm{y}$, or $\mathrm{z}$ directions) or $2^{\circ}$ (angular motion).

\section{ECM}

ECM of the pre-processed rsfMRI image data was performed using fast ECM (fECM) software ${ }^{4}$ (Wink et al., 2012). EC measures the sum of centralities of the node's direct neighbors, which are produced by calculating linear correlations between the fMRI time series and estimating eigenvector at each iteration. Biologically, EC can reflect the global functional importance of each voxel in the brain and has been shown to compare favorably to other centrality measures (Joyce et al., 2010). A node with a higher EC value means greater connectedness with the rest part of the brain. Compared to traditional ECM calculation methods, the time and memory required for the fECM algorithm are very small, because it computes the matrix-vector products directly from the data, without explicitly storing the connectivity matrix.

\section{Statistical Analysis}

Demographic continuous data and neuropsychological scores were expressed as means \pm standard deviations. For imaging data, the correlations between eigenvector centralities of different brain regions and neuropsychological scores were identified by linear correlation analysis performed in SPM8, age and sex as covariates. For the multiple comparison correction, Gaussian random field (voxel-level $P<0.001$, cluster level $P<0.05$ ) was employed. To further control the type I error, the Bonferroni method was further conducted in the multi-cognitive scale level.

\section{RESULTS}

One-hundred and one early stage PD patients had rsfMRI data. Seventeen patients were excluded due to imaging quality (including predominant head motion or scans that had not covered the whole brain). Finally, 84 subjects were included for further analysis. In the included patients, two participants were lack of SDMT scores. The detailed demographic data and neuropsychological assessment scores were listed in Table $\mathbf{1}$.

ECM analysis showed that EC values in the left inferior occipital gyrus (IOG) and lingual gyrus were positively correlated with the scores of the LNS test (Figure 1, Table 2). The immediate

\footnotetext{
${ }^{2} \mathrm{http}: / /$ rfmri.org/dpabi

${ }^{3} \mathrm{http}: / /$ www.fil.ion.ucl.ac.uk/spm

${ }^{4}$ https://github.com/amwink/bias/tree/master/matlab/fastECM
}

TABLE 1 | Demographic and neuropsychological data of Parkinson's disease

(PD) patients.

PD patients $(n=84)$

Female/male

$28 / 56$

Age (years)

$28 / 56$

Education (years)

UPDRS (motor scores)

$\mathrm{H}-\mathrm{Y}$ stage

HVLT-R

Immediate recall

Delayed recall

Retention

Recognition

BJLO

LNS

SF

SDMT
$15.21 \pm 3.01$

$1.71 \pm 0.49$

$47.94 \pm 13.63$

$49.94 \pm 14.23$

$50.74 \pm 13.34$

$53.93 \pm 13.91$

$12.74 \pm 1.83$

$11.01 \pm 2.71$

$53.62 \pm 11.47$

$44.43 \pm 9.44^{*}$
$20.87 \pm 10.31$

Values are expressed as means \pm standard deviations or numbers of subjects. UPDRS, Unified Parkinson's Disease Rating Scale; H-Y stage: Hoehn and Yahr stage; HVLT-R, Hopkins Verbal Learning Test-Revised; BJLO, Benton Judgment of Line Orientation test; LNS, WMS-III Letter Number Sequencing test; SF, Semantic Fluency test; SDMT, Symbol Digit Modalities Test. *SDMT scores were calculated from 82 patients.

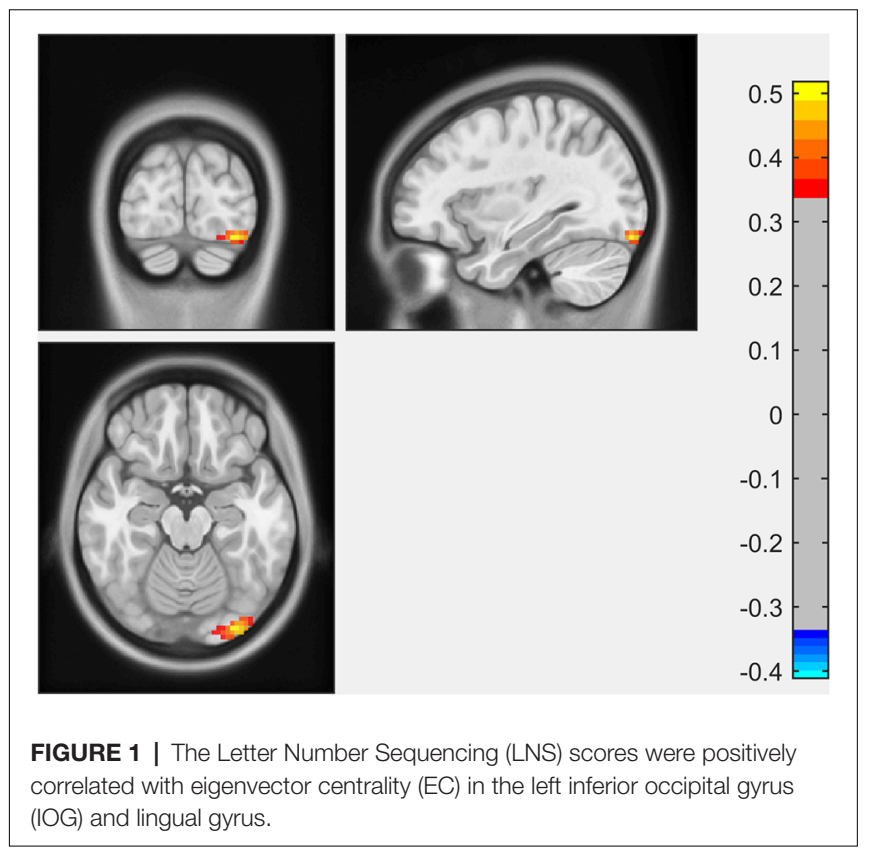

recall scores of HVLT-R were positively correlated with EC in the left superior frontal gyrus (SFG; Figure 2, Table 2). However, no significant correlations were detected between the scores of other cognitive assessments (delayed recall, retention, and recognition of HVLT-R, BJLO, SF, and SDMT) and the EC values.

After controlling the type I error at the multi-cognitive scale level, no significant correlation between EC values and cognitive scores was observed.

\section{DISCUSSION}

In the present study, we investigated the relationship between the performance of multiple cognitive tests and the eigenvector centrality of the whole brain. The major findings were as follows: 
TABLE 2 | Eigenvector centrality (EC) of brain areas showing significant correlations with neuropsychological scores

\begin{tabular}{|c|c|c|c|c|c|c|c|}
\hline \multirow[t]{2}{*}{ Cognitive scales } & \multirow[t]{2}{*}{ Cluster voxels } & \multirow[t]{2}{*}{ Brain regions } & \multirow[t]{2}{*}{ Peak intensity } & \multicolumn{3}{|c|}{ MNI coordinate } & \multirow[t]{2}{*}{$T$-value } \\
\hline & & & & $x$ & $y$ & $z$ & \\
\hline LNS & 57 & $\begin{array}{l}\text { Left inferior occipital gyrus } \\
\text { Left lingual gyrus }\end{array}$ & 0.51833 & -33 & -90 & -18 & 5.42 \\
\hline HVLT-R (immediate recall) & 104 & Left superior frontal gyrus & 0.41753 & -15 & 27 & 57 & 4.11 \\
\hline
\end{tabular}

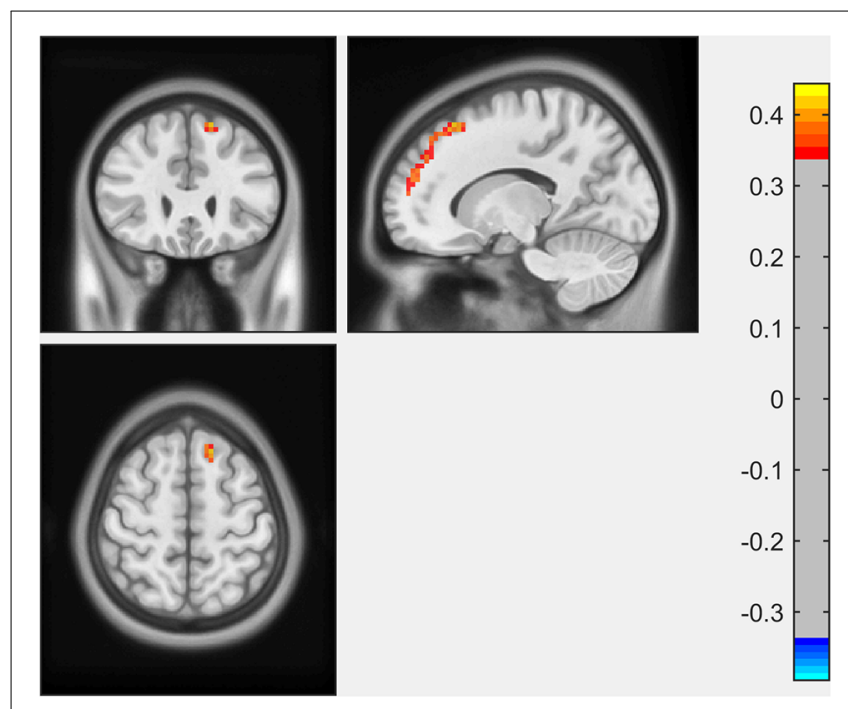

FIGURE 2 | The immediate recall scores of Hopkins Verbal Learning Test-Revised (HVLT-R) were positively correlated with EC in the left superior frontal gyrus (SFG).

(1) performance of working memory (LNS scores) in early PD was positively associated with EC in the left inferior occipital gyrus and lingual gyrus; and (2) EC in the left superior frontal gyrus was associated with immediate recall scores for memory in early PD patients. These results suggested that in the early stage of $\mathrm{PD}$, subtle alternations in different cognitive aspects could be reflected by the disruptions of functional connectivity using ECM.

To our knowledge, this is the first study using the ECM method to evaluate the whole brain network connectivity correlated with cognitive functions in the early stage PD patients. There were only a few studies that used ECM to investigate the underlying functional network alternations associated with motor and other nonmotor symptoms (e.g., depression, dysphagia) in PD patients (Holiga et al., 2015; Lou et al., 2015; Mueller et al., 2018; de Schipper et al., 2018; Gao et al., 2019). In a study by de Schipper et al. (2018), decreased EC was shown in the occipital and frontal lobes in PD patients, which indicated diminished connectivity of these regions within the overall brain functional architecture in PD.

The Letter-Number Sequencing Test is a measure of verbal working memory. Working memory, which refers to the ability to flexibly maintain and manipulate information for a short period, is generally considered a core component of executive function (Matthews, 2015). Our study showed a positive correlation between the LNS scores with the EC in the left occipital lobe (inferior occipital gyrus, lingual gyrus), which might imply an important role of these brain regions in the working memory performance in the early PD. There was growing evidence suggesting the posterior parts of the brain, including the occipital lobe, as the key pathophysiologic regions in cognitive impairment in PD. A positron emission tomography (PET) study showed decreased metabolism in the occipitoparietal cortex was associated with cognitive dysfunction in PD (Bohnen et al., 2011). Several MRI studies showed structural atrophy (Pagonabarraga et al., 2013; Garcia-Diaz et al., 2014; Pereira et al., 2014; Wu et al., 2018; Xuan et al., 2019) and functional disruption (Olde Dubbelink et al., 2014; Fang et al., 2017; Kawabata et al., 2018) in the occipital lobe in PD patients, which might contribute to impairment in multiple cognitive functions in PD (Pagonabarraga et al., 2013; Garcia-Diaz et al., 2014; Pereira et al., 2014). Disrupted functional connectivity of the occipital region was also reported in other neurodegenerative diseases, such as Lewy body dementia and Alzheimer's disease, suggesting a shared profile of functional changes in the cognitive disorders (Galvin et al., 2011). Conventionally, the cortical substrate for working memory/executive function tasks was predominantly attributed to the frontal region. However, working memory/executive function consisted of complex cognitive processes, and depended on distributed neural systems containing multiple brain regions (Petrides, 2015). Therefore, the term working memory is not necessarily equated with frontal function. In a task fMRI study, Ekman et al. (2014) found a significant activity decrease in the fusiform gyrus (a region adjacent to the lingual gyrus) during a verbal workingmemory task between baseline and follow-up in PD with MCI, indicating that the fusiform gyrus could be responsible for the longitudinal changes of working-memory maintenance in PD patients. Similar to our results, Lee et al. (2013) found that the performance of executive function task significantly correlated with occipital gray matter volume in early non-demented PD patients, rather than with the expected frontal region. The mechanisms of relationship between changes in the occipital lobe and cognitive decline in PD still need further investigation. Molecular imaging studies suggested that the degeneration of basal forebrain cholinergic fibers projecting to the posterior parts seemed to be an important cause for PD cognitive progression (Roy et al., 2016).

Another finding in our study was the positive correlation between EC of left SFG and immediate recall scores for memory. Previous studies showed the structural and functional changes of the frontal lobe in the early stages of PD, especially SFG and middle frontal gyrus (MFG). In a recent meta-analysis 
of structural studies in PD, left SFG was among the brain regions with the most significant regional gray matter volume reductions ( $\mathrm{Xu}$ et al., 2020). Generally, the temporal lobe is considered critical to the memory process, particularly episodic memory formation. However, damage in areas interconnected to the temporal lobe may also lead to memory impairment (Matthews, 2015). The frontal cortex receives inputs from all association areas including the ventral temporal lobe and limbic system (Ceccarini et al., 2019). Therefore, it also takes an important part in memory, mainly in encoding and retrieval functions. Lebedev et al. (2014) investigated rsfMRI correlate of cognitive impairment in $\mathrm{PD}$ patients from a large-scale network perspective. The result showed that better memory performance correlated with increased prefrontallimbic processing. In a rsfMRI study of rigidity-dominant PD, memory scores were positively correlated with the functional connectivity between the left inferior parietal lobule (IPL) and bilateral SFG (Hou et al., 2018). The neurotransmitter basis underlying changes of frontal areas in PD was thought to be related to depleted dopaminergic circuitry. However, more and more non-dopaminergic mechanisms, such as cholinergic, serotonergic and endocannabinoid systems (Cho et al., 2017; Ceccarini et al., 2019), were reported to participate in the frontal dysfunction, which might be responsible for declines in cognitive performance in early stage of PD. Moreover, we observed a dissociation in the EC correlation with different memory subtests. A previous structural study also showed that the immediate recall scores, but not the delayed recall scores, correlated with cortical thickness in PD patients (Pereira et al., 2014). It was in keeping with the finding that memory dysfunction in early PD patients to a large degree was a deficit of immediate recall (encoding), but not of retrieval or retention (Brønnick et al., 2011).

In our present study, there was no significant correlation between the performance of other cognitive assessments (BJLO, $\mathrm{SF}$, and SDMT) with EC. This might be partly due to PD patients in the current study being in an early disease stage with an only subtle decline in these cognitive functions. It is worth noting that the observed relationships between EC and working memory and immediate recall memory performance did not survive after Bonferroni adjustment in the multi-cognitive scale level. Probably, the relatively small sample size and only a few features taken into analysis in the present study might be the reason, and on other hand, the risk of high type II error might occur. As stated by historical document, the balance between type I and type II error should be paid attention to, instead of strictly controlling type I error, because type I error could be gradually eliminated when the scientific issues were widely answered and replicated by other researchers(Lieberman and Cunningham, 2009). Taken together, our explorative study, demonstrating the preliminary but important evidence for the potential coupling between brain EC alterations and neuropsychological behaviors, should help understand brain function topology during a cognitive performance in early PD patients.

Several limitations of our study should be recognized and further discussed. First, we did not include normal controls in the recent study. In the PPMI project, rsfMRI scanning was conducted at some selected sites. Therefore, only a small population of normal controls had rsfMRI images in the PPMI study $(n=22)$. Second, the neurological scales used in our study were not comprehensive enough. For example, we did not assess the language function because of the lack of specific language tests in the PPMI. Meanwhile, there are various aspects of executive functioning and memory, but our cognitive batteries only involved some specific components. Furthermore, we did not conduct a longitudinal study of fMRI data and cognitive performance. Future longitudinal studies with a larger population and combining multiple imaging modalities should be conducted to confirm our findings.

\section{CONCLUSIONS}

In summary, our study indicates that early brain functional alternations may account for the performance of different cognitive functions in early PD patients by using ECM analysis. The left occipital lobe (inferior occipital and lingual gyrus) is related to the performance of working memory. While the left superior frontal gyrus may contribute to a deficit in immediate recall of memory.

\section{DATA AVAILABILITY STATEMENT}

All datasets presented in this study are included in the article.

\section{ETHICS STATEMENT}

The studies involving human participants were reviewed and approved by ethics committee on human experimentation of each participating PPMI site (https://www.ppmi-info.org/aboutppmi/ppmi-clinical-sites/). Data used in the preparation of this article were obtained from the Parkinson's Progression Markers Initiative (PPMI) database (www.ppmi-info.org/data). The patients/participants provided their written informed consent to participate in this study.

\section{AUTHOR CONTRIBUTIONS}

FC and JZ designed the study and wrote the first draft of the article. XG analyzed the MRI data. YM and YS assisted with interpretation of findings. All authors contributed to the article and approved the submitted version.

\section{FUNDING}

Data used in the preparation of this article were obtained from the Parkinson's Progression Markers Initiative (PPMI) database (www.ppmi-info.org/data). For up-to-date information on the study, visit (www.ppmiinfo.org). PPMI-a public-private partnership-is funded by the Michael J. Fox Foundation for Parkinson's Research and funding partners, including AbbVie, Allergan, Amathus Therapeutics, Avid Radiopharmaceuticals, Biogen, BioLegend, Bristol-Myers Squibb, Celgene, Denali, GE 
Healthcare, Genentech, GlaxoSmithKline (GSK), Golub Capital, Handl Therapeutics, Insitro, Janssen Neuroscience, Eli Lilly and Company, Lundbeck, Merck, Meso Scale Discovery (MSD), Pfizer, Piramal Imaging, Prevail Therapeutics, Roche, Sanofi Genzyme, Servier, Takeda, Teva, UCB, Verily, and Voyager Therapeutics (www.ppmi-info.org/fundingpartners). This work was also supported by the Natural Science Foundation of Zhejiang Province (Grant No. LY17H090020). XG is supported

\section{REFERENCES}

Aarsland, D., Creese, B., Politis, M., Chaudhuri, K. R., Ffytche, D. H., Weintraub, D., et al. (2017). Cognitive decline in Parkinson disease. Nat. Rev. Neurol. 13, 217-231. doi: 10.1038/nrneurol.2017.27

Agosta, F., Canu, E., Stefanova, E., Sarro, L., Tomić, A., šica, V., et al. (2014). Mild cognitive impairment in Parkinson's disease is associated with a distributed pattern of brain white matter damage. Hum. Brain Mapp. 35, 1921-1929. doi: 10.1002/hbm.22302

Baggio, H.-C., Sala-Llonch, R., Segura, B., Marti, M.-J., Valldeoriola, F., Compta, Y., et al. (2014). Functional brain networks and cognitive deficits in Parkinson's disease. Hum. Brain Mapp. 35, 4620-4634. doi: 10.1002/hbm. 22499

Binnewijzend, M. A. A., Adriaanse, S. M., Van der Flier, W. M., Teunissen, C. E., de Munck, J. C., Stam, C. J., et al. (2014). Brain network alterations in Alzheimer's disease measured by eigenvector centrality in fMRI are related to cognition and CSF biomarkers. Hum. Brain Mapp. 35, 2383-2393. doi: 10.1002/hbm. 22335

Bohnen, N. I., Koeppe, R. A., Minoshima, S., Giordani, B., Albin, R. L., Frey, K. A., et al. (2011). Cerebral glucose metabolic features of Parkinson disease and incident dementia: longitudinal study. J. Nucl. Med. 52, 848-855. doi: 10.2967/jnumed.111.089946

Brønnick, K., Alves, G., Aarsland, D., Tysnes, O.-B., and Larsen, J. P. (2011). Verbal memory in drug-naive, newly diagnosed Parkinson's disease: the retrieval deficit hypothesis revisited. Neuropsychology 25, 114-124. doi: $10.1037 / \mathrm{a} 0020857$

Ceccarini, J., Casteels, C., Ahmad, R., Crabbé, M., Van de Vliet, L., Vanhaute, H., et al. (2019). Regional changes in the type 1 cannabinoid receptor are associated with cognitive dysfunction in Parkinson's disease. Eur. J. Nucl. Med. Mol. Imaging 46, 2348-2357. doi: 10.1007/s00259-019-04445-X

Cho, S. S., Strafella, A. P., Duff-Canning, S., Zurowski, M., Vijverman, A.-C., Bruno, V., et al. (2017). The relationship between serotonin-2A receptor and cognitive functions in nondemented Parkinson's disease patients with visual hallucinations. Mov. Disord. Clin. Pract. 4, 698-709. doi: 10.1002/mdc3. 12466

de Schipper, L. J., Hafkemeijer, A., van der Grond, J., Marinus, J., Henselmans, J. M. L., and van Hilten, J. J. (2018). Altered whole-brain and network-based functional connectivity in Parkinson's disease. Front. Neurol. 9:419. doi: 10.3389/fneur.2018.00419

Díez-Cirarda, M., Strafella, A. P., Kim, J., Pena, J., Ojeda, N., CabreraZubizarreta, A., et al. (2018). Dynamic functional connectivity in Parkinson's disease patients with mild cognitive impairment and normal cognition. NeuroImage Clin. 17, 847-855. doi: 10.1016/j.nicl.2017. 12.013

Ekman, U., Eriksson, J., Forsgren, L., Domellof, M. E., Elgh, E., Lundquist, A., et al. (2014). Longitudinal changes in task-evoked brain responses in Parkinson's disease patients with and without mild cognitive impairment. Front. Neurosci. 8:207. doi: 10.3389/fnins.2014.00207

Fang, J., Chen, H., Cao, Z., Jiang, Y., Ma, L., Ma, H., et al. (2017). Impaired brain network architecture in newly diagnosed Parkinson's disease based on graph theoretical analysis. Neurosci. Lett. 657, 151-158. doi: 10.1016/j.neulet.2017. 08.002

Galvin, J. E., Price, J. L., Yan, Z., Morris, J. C., and Sheline, Y. I. (2011). Resting bold fMRI differentiates dementia with Lewy bodies vs. Alzheimer disease. Neurology 76, 1797-1803. doi: 10.1212/WNL.0b013e31821ccc83 by the China Postdoctoral Science Foundation (Grant No. 2019M662082).

\section{ACKNOWLEDGMENTS}

We would like to thank Dr. Junjie $\mathrm{Wu}$, from the Clinical Research Institute of Zhejiang Provincial People's Hospital, for his contributions to editing the grammar issues.

Gao, J., Guan, X., Cen, Z., Chen, Y., Ding, X., Lou, Y., et al. (2019). Alteration of brain functional connectivity in Parkinson's disease patients with dysphagia. Dysphagia 34, 600-607. doi: 10.1007/s00455-019-10015-y

Gao, Y., Nie, K., Huang, B., Mei, M., Guo, M., Xie, S., et al. (2017). Changes of brain structure in Parkinson's disease patients with mild cognitive impairment analyzed via VBM technology. Neurosci. Lett. 658, 121-132. doi: 10.1016/j. neulet.2017.08.028

Garcia-Diaz, A. I., Segura, B., Baggio, H. C., Marti, M. J., Valldeoriola, F., Compta, Y., et al. (2014). Structural MRI correlates of the MMSE and pentagon copying test in Parkinson's disease. Parkinsonism Relat. Disord. 20, 1405-1410. doi: 10.1016/j.parkreldis.2014.10.014

Gerrits, N. J. H. M., van Loenhoud, A. C., van den Berg, S. F., Berendse, H. W., Foncke, E. M. J., Klein, M., et al. (2016). Cortical thickness, surface area and subcortical volume differentially contribute to cognitive heterogeneity in Parkinson's disease. PLoS One 11:e0148852. doi: 10.1371/journal.pone. 0148852

Holiga, S., Mueller, K., Möller, H. E., Urgošík, D., Ružička, E., Schroeter, M. L., et al. (2015). Resting-state functional magnetic resonance imaging of the subthalamic microlesion and stimulation effects in Parkinson's disease: indications of a principal role of the brainstem. NeuroImage Clin. 9, 264-274. doi: 10.1016/j.nicl.2015.08.008

Hou, Y., Yang, J., Luo, C., Ou, R., Zou, Y., Song, W., et al. (2018). Restingstate network connectivity in cognitively unimpaired drug-naive patients with rigidity-dominant Parkinson's disease. J. Neurol. Sci. 395, 147-152. doi: 10.1016/j.jns.2018.10.003

Joyce, K. E., Laurienti, P. J., Burdette, J. H., and Hayasaka, S. (2010). A new measure of centrality for brain networks. PLoS One 5:e12200. doi: 10.1371/journal.pone. 0012200

Kawabata, K., Watanabe, H., Hara, K., Bagarinao, E., Yoneyama, N., Ogura, A., et al. (2018). Distinct manifestation of cognitive deficits associate with different resting-state network disruptions in non-demented patients with Parkinson's disease. J. Neurol. 265, 688-700. doi: 10.1007/s00415-0188755-5

Lee, J. E., Park, H. J., Song, S. K., Sohn, H. H., Lee, J. D., and Lee, P. H. (2010). Neuroanatomic basis of amnestic MCI differs in patients with and without Parkinson disease. Neurology 75, 2009-2016. doi: 10.1212/WNL.0b013e3181ff96bf

Lee, E.-Y., Sen, S., Eslinger, P. J., Wagner, D., Shaffer, M. L., Kong, L., et al. (2013). Early cortical gray matter loss and cognitive correlates in non-demented Parkinson's patients. Parkinsonism Relat. Disord. 19, 1088-1093. doi: 10.1016/j. parkreldis.2013.07.018

Lebedev, A. V., Westman, E., Simmons, A., Lebedeva, A., Siepel, F. J., Pereira, J. B., et al. (2014). Large-scale resting state network correlates of cognitive impairment in Parkinson's disease and related dopaminergic deficits. Front. Syst. Neurosci. 8:45. doi: 10.3389/fnsys.2014.00045

Lieberman, M. D., and Cunningham, W. A. (2009). Type I and type II error concerns in fMRI research: re-balancing the scale. Soc. Cogn. Affect. Neurosci. 4, 423-428. doi: 10.1093/scan/nsp052

Lohmann, G., Margulies, D. S., Horstmann, A., Pleger, B., Lepsien, J., Goldhahn, D., et al. (2010). Eigenvector centrality mapping for analyzing connectivity patterns in fMRI data of the human brain. PLoS One 5:e10232. doi: 10.1371/journal.pone.0010232

Lou, Y., Huang, P., Li, D., Cen, Z., Wang, B., Gao, J., et al. (2015). Altered brain network centrality in depressed Parkinson's disease patients. Mov. Disord. 30, 1777-1784. doi: 10.1002/mds.26321 
Matthews, B. R. (2015). Memory dysfunction. Continuum 21, 613-626. doi: 10.1212/01.con.0000466656.59413.29

Melzer, T. R., Watts, R., MacAskill, M. R., Pitcher, T. L., Livingston, L., Keenan, R. J., et al. (2012). Grey matter atrophy in cognitively impaired Parkinson's disease. J. Neurol. Neurosurg. Psychiatry 83, 188-194. doi: 10.1136/jnnp-2011-300828

Monchi, O., Petrides, M., Mejia-Constain, B., and Strafella, A. P. (2007). Cortical activity in Parkinson's disease during executive processing depends on striatal involvement. Brain 130, 233-244. doi: 10.1093/brain/awl326

Mueller, K., Jech, R., Ružička, F., Holiga, š Ballarini, T., Bezdicek, O., et al. (2018). Brain connectivity changes when comparing effects of subthalamic deep brain stimulation with levodopa treatment in Parkinson's disease. NeuroImage Clin. 19, 1025-1035. doi: 10.1016/j.nicl.2018.05.006

Olde Dubbelink, K. T. E., Schoonheim, M. M., Deijen, J. B., Twisk, J. W., Barkhof, F., and Berendse, H. W. (2014). Functional connectivity and cognitive decline over 3 years in Parkinson disease. Neurology 83, 2046-2053. doi: 10.1212/wnl.0000000000001020

Pagonabarraga, J., and Kulisevsky, J. (2012). Cognitive impairment and dementia in Parkinson's disease. Neurobiol. Dis. 46, 590-596. doi: 10.1016/j.nbd.2012. 03.029

Pagonabarraga, J., Corcuera-Solano, I., Vives-Gilabert, Y., Llebaria, G., GarciaSanchez, C., Pascual-Sedano, B., et al. (2013). Pattern of regional cortical thinning associated with cognitive deterioration in Parkinson's disease. PLoS One 8:e54980. doi: 10.1371/journal.pone.0054980

Pereira, J. B., Svenningsson, P., Weintraub, D., Brønnick, K., Lebedev, A., Westman, E., et al. (2014). Initial cognitive decline is associated with cortical thinning in early Parkinson disease. Neurology 82, 2017-2025. doi: 10.1212/wnl.0000000000000483

Petrides, M. (2015). "Working memory, neural basis of," in International Encyclopedia of the Social and Behavioral Sciences, ed. J. D. Wright, 2nd Edn. (Oxford: Elsevier), 703-710.

Roy, R., Niccolini, F., Pagano, G., and Politis, M. (2016). Cholinergic imaging in dementia spectrum disorders. Eur. J. Nucl. Med. Mol. Imaging 43, 1376-1386. doi: 10.1007/s00259-016-3349-x

Shen, Z., Huang, P., Wang, C., Qian, W., Yang, Y., and Zhang, M. (2017). Increased network centrality as markers of relapse risk in nicotine-dependent individuals treated with varenicline. Prog. Neuropsychopharmacol. Biol. Psychiatry 75, 142-147. doi: 10.1016/j.pnpbp.2017.02.002

Song, Z., Zhang, M., and Huang, P. (2016). Aberrant emotion networks in early major depressive disorder patients: an eigenvector centrality mapping study. Transl. Psychiatry 6:e819. doi: 10.1038/tp.2016.81
Tessitore, A., Esposito, F., Vitale, C., Santangelo, G., Amboni, M., Russo, A., et al. (2012). Default-mode network connectivity in cognitively unimpaired patients with Parkinson disease. Neurology 79, 2226-2232. doi: 10.1212/WNL. 0b013e31827689d6

Williams-Gray, C. H., Foltynie, T., Brayne, C. E. G., Robbins, T. W., and Barker, R. A. (2007). Evolution of cognitive dysfunction in an incident Parkinson's disease cohort. Brain 130, 1787-1798. doi: 10.1093/brain/ awm111

Wink, A. M., de Munck, J. C., van der Werf, Y. D., van den Heuvel, O. A., and Barkhof, F. (2012). Fast eigenvector centrality mapping of voxel-wise connectivity in functional magnetic resonance imaging: implementation, validation and interpretation. Brain Connect. 2, 265-274. doi: 10.1089/brain. 2012.0087

Wu, Q., Gao, Y., Liu, A.-S., Xie, L.-Z., Qian, L., and Yang, X.-G. (2018). Large-scale cortical volume correlation networks reveal disrupted small world patterns in Parkinson's disease. Neurosci. Lett. 662, 374-380. doi: 10.1016/j.neulet.2017. 10.032

Xu, X., Han, Q., Lin, J., Wang, L., Wu, F., and Shang, H. (2020). Grey matter abnormalities in Parkinson's disease: a voxel-wise meta-analysis. Eur. J. Neurol. 27, 653-659. doi: 10.1111/ene.14132

Xuan, M., Guan, X., Huang, P., Shen, Z., Gu, Q., Yu, X., et al. (2019). Different patterns of gray matter density in early- and middle-late-onset Parkinson's disease: a voxel-based morphometry study. Brain Imaging Behav. 13, 172-179. doi: 10.1007/s11682-0179745-4

Yarnall, A. J., Breen, D. P., Duncan, G. W., Khoo, T. K., Coleman, S. Y., Firbank, M. J., et al. (2014). Characterizing mild cognitive impairment in incident Parkinson disease: the ICICLE-PD study. Neurology 82, 308-316. doi: $10.1212 / \mathrm{wnl} .0000000000000066$

Conflict of Interest: The authors declare that the research was conducted in the absence of any commercial or financial relationships that could be construed as a potential conflict of interest.

Copyright (c) 2020 Cao, Guan, Ma, Shao and Zhong. This is an open-access article distributed under the terms of the Creative Commons Attribution License (CC BY). The use, distribution or reproduction in other forums is permitted, provided the original author(s) and the copyright owner(s) are credited and that the original publication in this journal is cited, in accordance with accepted academic practice. No use, distribution or reproduction is permitted which does not comply with these terms. 